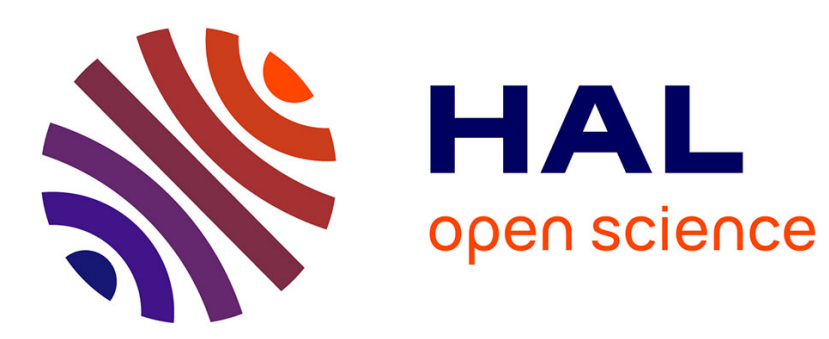

\title{
Changer sans perdre: le dilemme cornélien des élus locaux
}

\begin{abstract}
Alain Faure
\section{To cite this version:}

Alain Faure. Changer sans perdre: le dilemme cornélien des élus locaux. Revue française d'administration publique, 2012, 141, p. 99-107. halshs-00698934
\end{abstract}

\section{HAL Id: halshs-00698934 https://shs.hal.science/halshs-00698934}

Submitted on 18 May 2012

HAL is a multi-disciplinary open access archive for the deposit and dissemination of scientific research documents, whether they are published or not. The documents may come from teaching and research institutions in France or abroad, or from public or private research centers.
L'archive ouverte pluridisciplinaire HAL, est destinée au dépôt et à la diffusion de documents scientifiques de niveau recherche, publiés ou non, émanant des établissements d'enseignement et de recherche français ou étrangers, des laboratoires publics ou privés. 


\title{
CHANGER SANS PERDRE : LE DILEMME CORNÉLIEN DES ÉLUS LOCAUX
}

\author{
Alain Faure
}

E.N.A. | Revue française d'administration publique

$2012 / 1-n^{\circ} 141$

pages 99 à 107

ISSN 0152-7401

Article disponible en ligne à l'adresse:

http://www.cairn.info/revue-francaise-d-administration-publique-2012-1-page-99.htm

Pour citer cet article :

Faure Alain, « Changer sans perdre : le dilemme cornélien des élus locaux »,

Revue française d'administration publique, 2012/1 n¹41, p. 99-107. DOI : 10.3917/rfap.141.0099

Distribution électronique Cairn.info pour E.N.A.

(C) E.N.A.. Tous droits réservés pour tous pays.

La reproduction ou représentation de cet article, notamment par photocopie, n'est autorisée que dans les limites des conditions générales d'utilisation du site ou, le cas échéant, des conditions générales de la licence souscrite par votre établissement. Toute autre reproduction ou représentation, en tout ou partie, sous quelque forme et de quelque manière que ce soit, est interdite sauf accord préalable et écrit de l'éditeur, en dehors des cas prévus par la législation en vigueur en France. II est précisé que son stockage dans une base de données est également interdit. 


\title{
DYNAMIQUES FONCTIONNELLES
}

\section{CHANGER SANS PERDRE : LE DILEMME CORNÉLIEN DES ÉLUS LOCAUX}

\author{
Alain FAURE \\ Directeur de recherche au CNRS, Université de Grenoble
}

\begin{abstract}
Résumé
Pourquoi et comment l'élan réformateur tant attendu de la loi du 16 décembre 2010 s'est-il si rapidement trouvé en tension, et parfois en décalage frontal, avec les représentations partagées du changement qu'il a pu suscitées initialement ? L'analyse portera essentiellement sur la prise de position des élus locaux dans ces controverses : en changeant d'aire, les élus locaux redoutent de « perdre la face » dans les joutes politiques locales ; en changeant d'ère, ils craignent que leur commune perde son âme et son identité collective. Ces deux lignes narratives donnent des indications précieuses sur les sentiers de dépendance qui balisent les représentations du politique et du changement dans l'action publique locale.
\end{abstract}

\section{Mots-clefs}

Récit, frontière, langage, pouvoir, identité

\section{Abstract}

- Implementing Change without Detrimental Consequences: The Cornelian dilemma faced by Local Elected Representatives - There was a great deal of momentum for reform with the long-awaited law of 16 December 2010. How and why did this initial fervour for reform so quickly wane and sometimes enter into direct conflict with the shared ideas for change aroused early on? This analysis mainly addresses the stance taken in this controversy by local-level elected representatives. They feel, on the one hand, that implementing these changes will cause them to "lose face" in the local political arena, and on the other, that their cities may lose their soul and their collective identity. These two narratives provide precious insight into the dependence created by images of politics and change in local government action.

\section{Keywords}

| Narrative, border, language, power, identity

Dans tout affichage de réforme, on trouve l'énoncé d'un "grand récit » sur le bien commun et sur l'intérêt général. Concernant la loi du 16 décembre 2010, la communication gouvernementale dévoile les grandes lignes de ce récit dans un fascicule de 
douze pages ${ }^{1}$ qui place en exergue une citation fervente du Président de la République : «Cette réforme stimule l'initiative et les énergies locales. Elle renforce les libertés locales, celles dont Tocqueville disait qu'elles sont la force des peuples libres ». Sous-titrée « une nouvelle impulsion pour les territoires », la brochure organise sa première page avec l'affichage coloré de noms communs (financement, coopération, synergie, collectivités, intercommunalité, élections) et de verbes (simplifier, alléger, clarifier), autant de mots choisis pour imager le souffle et les orientations de la réforme. Dans la présente étude, nous souhaitons chercher à mieux comprendre pourquoi et comment cet élan réformateur tant attendu s'est si rapidement trouvé en tension, et parfois en décalage frontal, avec les représentations partagées du changement qu'il a pu suscitées initialement. En effet, dans les multiples rapports qui ont précédé la loi et dans les grandes priorités qui en structurent le contenu, on trouve une série de diagnostics qui faisaient apparemment l'objet d'un large consensus dans les arènes partisanes et administratives, aussi bien sur le versant de la critique (revoir des découpages cantonaux archaïques, une fiscalité désuète, des empilements institutionnels opaques et contre-productifs) que sur celui des grands plaidoyers vertueux (mieux penser la solidarité, la proximité, les péréquations, la démocratie locale). Le fait de réviser les règles du jeu de la décentralisation semblait validé sur quelques objectifs robustes : des intercommunalités urbaines plus efficaces, des élus communautaires au suffrage universel direct, une régulation publique métropolitaine plus cohérente, une meilleure coordination des services publics aux échelons régional et national. Pourtant, au fur et à mesure des annonces gouvernementales, des voix se sont élevées pour contester tout à la fois la philosophie, les instruments et les finalités de la réforme. Si la plupart des observateurs de la réforme décortiquent les données techniques, juridiques et institutionnelles qui pourraient expliquer cette puissante levée de boucliers, ces analyses ne délivrent que peu d'informations sur la dimension sensible, symbolique, presque passionnelle qui a pu orienter les discours, durant cette période, sur les raisons de la colère et sur ce que devrait être une «bonne décentralisation».

Nous faisons l'hypothèse que cette piste de lecture est utile aux analystes dans la mesure où elle nous informe sur les conditions d'appréhension et de formulation des changements dans l'action publique locale. En proposant une incursion au cœur des controverses suscitées par le projet gouvernemental, nous souhaitons mettre en lumière les « lignes narratives » que les acteurs étudiés avancent pour prendre position sur le bienfondé des mesures annoncées et ainsi identifier d'éventuelles « coalitions discursives » emblématiques des mobilisations sociales sur ces enjeux institutionnels ${ }^{2}$. La réflexion s'inscrit dans la voie des travaux de science politique consacrés à la fabrique discursive des politiques publiques, sur l'idée que les mots du pouvoir sont, pour reprendre une belle formule foucaldienne, « une violence que nous faisons aux choses ». C'est l'hypothèse que l'activité politique se loge dans les activités discursives et que le langage joue un rôle décisif pour «maintenir un ordre face à une réalité toujours désordonnée, discontinue et insaisissable $»^{3}$. Si les idéaux tocquevilliens en faveur de la liberté et de l'initiative locales sont assurément au cœur de tout processus de décentralisation, toute la question est de savoir comment s'opère la cristallisation des idées vers cette supposée modernité politique.

1. http://www.interieur.gouv.fr/sections/reforme-collectivites.

2. Hajer (Maarten), Law (David), « Ordering through discourse », in Moran (Rein), Goodin (Robert), The Oxford Handbook of public Policy, Oxford University Press, 2006, p. 251-268.

3. Zittoun (Philippe), La fabrique politique des politiques publiques, Grenoble, Institut d'Etudes Politiques, Habilitation à Diriger des Recherches, 2011, 345 p. 
Les plaidoyers initiaux favorables à la réforme comme ceux qui l'ont par la suite vertement critiquée nous informent sur les processus complexes de désenchantement et de réenchantement du monde par le verbe, et nous faisons l'hypothèse que leur analyse permet de mieux comprendre l'interdépendance des représentations, des discours et des pratiques dans les champs imbriqués de la compétition politique et des politiques publiques.

Sur le plan méthodologique, notre analyse prend appui sur un matériau de témoignages recueillis entre janvier et juillet 2011. Ces données proviennent du suivi (parfois de l'animation) de dix débats publics et réunions de travail consacrés aux enjeux et à la portée de la réforme. Ces rencontres ont mobilisé des panels très diversifiés d'acteurs (élus, fonctionnaires, professionnels, habitants, experts...) et il faut souligner que leur intensité s'est fortement accrue à partir de mai 2011 (c'est-à-dire après que les préfets eurent annoncé leurs préconisations sur les périmètres intercommunaux). Nous avons aussi procédé à une série d'entretiens individualisés auprès d'élus locaux, de fonctionnaires territoriaux et d'experts qui étaient placés en première ligne dans la mise en œuvre de la loi.

L'analyse portera essentiellement sur la prise de position des élus locaux dans ces controverses et la réflexion sera focalisée sur deux questionnements qui sont apparus de façon récurrente dans les témoignages. Le premier porte directement sur la mise en application de la loi et sur le fait que le redimensionnement des intercommunalités pourrait entraîner une appréhension plus efficace des enjeux d'action publique (changer d'aire pour changer d'air ${ }^{4}$ ). Le second questionnement porte à l'inverse sur les dynamiques de gouvernance et de démocratie participative et sur l'influence de leur essor dans la définition du périmètre « pertinent » des collectivités intercommunales (c'est le fait de changer d'ère dans la façon d'appréhender les problèmes collectifs qui entraînerait une réflexion sur l'élargissement des aires intercommunales). Même si les deux idées sont entremêlées et imbriquées, il nous a semblé possible, au fil des figures de discours recueillies, de les mettre en équation pour éclairer les résistances au changement qu'elles illustraient : en changeant d'ère, les élus locaux redoutent de «perdre la face » dans les joutes politiques locales ; en changeant d'ère, ils craignent que leur commune perde son âme et son identité collective. Nous tenterons de montrer comment ces deux représentations de la réforme, dans la multiplicité des déclinaisons que les témoignages ont produit, donnent des indications précieuses sur les sentiers de dépendance ${ }^{5}$ qui balisent les représentations du politique et du changement dans l'action publique locale. L'enquête permet de souligner la dimension passionnelle et territorialisée de ces sentiers et elle met en évidence que l'idéal démocratique de la décentralisation (la fameuse force des peuples libres) place en fait les élus locaux face à un véritable dilemme cornélien.

\section{COMMENT CHANGER D'AIRE SANS « PERDRE LA FACE »?}

Jusqu'à ce que les préfets ne fassent des propositions concrètes à la commission de coopération intercommunale pour diminuer et rationnaliser découpages intercommunaux, la réforme suscitait des réactions contenues dans les cercles fermés de chaque microcosme politique local et presque exclusivement argumentées sur des motifs positionnels. Les élus

4. Cette formule a été choisie par la Communauté d'Agglomération Grenoble Alpes Métropole pour annoncer un débat public sur la réforme territoriale en juin 2011.

5. Pierson (Paul), "Increasing Returns, Path Dependence, and the Study of Politics », The American Political Science Review, n 94 , 2000, p. 251-267. 
municipaux, les conseillers généraux et les conseillers régionaux ont d'abord décrypté les initiatives gouvernementales sur la décentralisation à l'aune de calculs électoraux et financiers sur trois zones d'incertitudes : les effets de la suppression de la taxe professionnelle, l'élection des futurs conseillers territoriaux et la représentativité des conseillers communautaires dans les nouvelles structures intercommunales. Cette première façon d'appréhender le changement, qui n'est ni surprenante ni originale en soi, nous informe sur les combats premiers qui fondent l'engagement dans des responsabilités politiques locales. La conquête du pouvoir local par les urnes, les leviers (fiscaux et politiques) de préservation de l'autonomie territoriale et la formation des coalitions politico-administratives dans les assemblées départementales et régionales sont les trois principaux registres d'action qui permettent aux élus locaux d'assoir leur triple légitimité d'éligible, de notable et d'entrepreneur $^{6}$. Le dévoilement des préconisations préfectorales en mai 2011 a modifié la nature des débats dans deux directions inattendues : d'une part la réforme a été critiquée avec virulence pour sa dimension autoritaire, d'autre part les médias locaux ont explicitement évoqué dans chaque département les conflits de leadership intercommunal entrainés par les nouveaux découpages. Ce virage était moins prévisible que le combat positionnel de départ dans la mesure où il a soudain donné à la loi une visibilité publique et médiatique qu'aucune réforme de décentralisation n'avait jusqu'alors suscitée. En quelques semaines, des acteurs d'horizons très variés ont publiquement dénoncé le volontarisme de l'État, la place trop favorable accordée aux métropoles et la parole citoyenne confisquée.

Il faut ici souligner que les débats portaient jusqu'alors essentiellement sur d'autres enjeux. L'essentiel des controverses était centré sur quatre défis : deux grandes priorités (lutter contre les inégalités territoriales et promouvoir des régulations métropolitaines à la hauteur des défis de gestion publique en présence) et deux transformations (revoir le système électoral cantonal unanimement jugé archaïque et clarifier les responsabilités entre les départements et les régions). C'est sur ces quatre chantiers que les principales associations d'élus avaient construit leur doctrine de la réforme et c'est sur ces transformations du design institutionnel subnational que le consensus modernisateur initial semblait s'être établi.

Le début de l'année 2011 est caractérisé par un tournant narratif au cours duquel les enjeux de compétition politique et d'identité communale deviennent centraux. L'enquête permet de constater que les arguments positionnels avancés par les élus locaux gagnent rapidement du terrain, qu'ils sont relayés par les médias locaux et rapidement commentés dans différents espaces collectifs. Ces prises de position se caractérisent par un état d'esprit d'abord cristallisé sur l'idée qu'en changeant d'aire (cantonale et intercommunale), les élus locaux vont perdre la face dans les joutes politiques quotidiennes qui les opposent aux autres collectivités et à l'État. Les récits mettent en intrigue ce sentiment d'impuissance et de défaite autour de deux lignes discursives. La première concerne l'imagerie guerrière et héroïque des élus locaux qui défendent l'intérêt général à l'échelon municipal. Dans les propos recueillis, les maires et les conseillers généraux soulignent qu'ils tirent d'abord leur légitimité des rapports de force qu'ils parviennent à établir avec leurs voisins et avec les administrations. La seconde ligne discursive concerne les combats du monde rural pour résister aux menaces et aux dangers de l'expansion urbaine. Dès lors que leur commune se situe hors du premier cercle d'une agglomération, les élus locaux considèrent la ville comme un acteur hégémonique et adoptent une position de résistance

6. Il existe toute une littérature académique sur ces trois cœurs de métier de l'activité politique locale. Pour une synthèse, voir notamment : Fontaine (Joseph), Le Bart Christian), Le métier d'élu local, Paris, L'Harmattan, 2000 ; Faure (Alain), « Les apprentissages du métier d'élu local : la tribu, le système et les arènes », Pôle Sud, n ${ }^{\circ}$, novembre 1997, p. 72-79. 
et de repli. C'est l'idée que la «métropole », telle qu'elle est définie dans la loi, véhicule une représentation de l'action publique porteuse de domination, d'annexion et de mépris. Il faut noter que cette appréhension concurrentielle et inégalitaire du dialogue interterritorial se greffe, par un effet de contexte, à la perception d'un État libéral qui serait favorable aux grandes villes et qui prendrait ses distances avec les préoccupations du monde rural.

Sur le plan symbolique, ces deux figures de discours entrent en écho avec une imagerie classique de l'identité française. On en trouve trace par exemple dans les propos d'un expert $^{7}$ qui évoque avec malice lors des débats La guerre des boutons ${ }^{8}$ entre les petites communes. Dans ce roman célèbre situé au XIX ${ }^{\mathrm{e}}$ siècle, l'agressivité et l'obstination des enfants de Longeverne et de Verlans sont légitimes, à défaut d'être justifiées, dès lors que cette dynamique de groupe est adossée à un système de valeurs promouvant la solidarité, la fierté et la vaillance. Le trophée des boutons (dérobés sur les vêtements de la bande rivale) rappelle celui des entreprises et des résidents que les intercommunalités cherchent en permanence à capter sur des territoires mitoyens. L'obsession de ne pas perdre la face relève certes de l'orgueil et de la fierté du groupe mais c'est aussi l'enjeu du lien social qui est posé par cette attitude : le collectif est là pour assurer en même temps la protection et la reconnaissance de ses membres, il permet d'articuler les raisonnements du « je peux compter sur » et du $\left\langle\right.$ je compte pour $»^{9}$. Dans un autre débat public, un élu local fait référence à la fable de La Fontaine sur le pot de fer contre le pot de terre, déplorant que les mondes rural et urbain rejouent avec la réforme la scène finale lorsque, enfin, un voyage commun est programmé associant le pot de fer et le pot de terre :

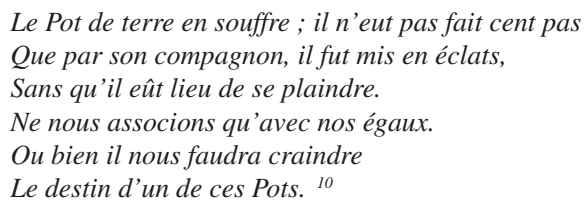

Les prises de position montrent clairement que les élus locaux revendiquent leur légitimité sur leur capacité à dialoguer d'égal à égal avec leurs voisins, fussent-ils puissants. Le sentiment de «perdre la face » dans la période de discussion sur le redécoupage des périmètres intercommunaux est exprimé sur le mode de l'inquiétude et de la colère. C'est ainsi qu'un député périurbain (et président d'une structure intercommunale) s'exclame pour commenter les préconisations préfectorales : « Si j'ai bien compris la réforme, le Gouvernement et les métropoles ne nous laissent le choix qu'entre nous soumettre ou rester jouer dans un bac à sable ».

\section{COMMENT CHANGER D'ÈRE SANS PERDRE SON ÂME?}

Les réactions combatives des élus locaux sur la réforme illustrent d'abord les ressorts cognitifs classiques de la compétition politique dans son ancrage socio-historique. On pense en premier lieu aux travaux sur l'empreinte des sentiers institutionnels ${ }^{11}$ qui

7. Propos avancé dans un débat public sur la réforme par le politiste Emmanuel Négrier.

8. Pergaud (Louis), La guerre des boutons, roman de ma douzième année, Editions Mercure de France, 1912.

9. Hypothèse évoquée dans un débat public sur la réforme par le sociologue Serge Paugam.

10. La Fontaine (Jean), Les fables choisies, Librairie Coulet, 1668.

11. Pierson, op. cit. 
orientent les cartes mentales des individus : les discours reflètent et entretiennent en effet une représentation du pouvoir puissamment attachée aux fondations communaliste et départementaliste du système politique français. Cependant, l'étude des commentaires suscités par l'annonce des préconisations préfectorales permet aussi d'identifier des facettes de la compétition politique qui portent moins sur la défense d'intérêts et d'acquis que sur l'expression passionnelle de valeurs et d'idéaux. Ce constat doit être pris au sérieux dans la mesure où il donne des informations nouvelles par rapport aux travaux récents consacrés à l'émergence des idéologies territoriales ${ }^{12}$ et aux nouvelles grammaires de l'action publique locale ${ }^{13}$. Ces deux ouvrages collectifs ont cherché à mieux décrypter, sur le cas français et depuis deux décennies, la progression des discours plutôt techniques et gestionnaires qui accompagnent les pratiques de gouvernance territoriale et de management public local. Les auteurs proposent un travail de dévoilement sur les lexiques des acteurs locaux en matière de développement, d'excellence, de performance, de gouvernance, de durabilité, de projet, de citoyenneté. Malgré la diversité de leurs objets, les enquêtes insistent toutes sur la place structurante que les enjeux de professionnalisation jouent dorénavant dans les apprentissages de l'action publique locale. Et les auteurs soulignent en conclusion que ce sont d'abord les expertises catégorielles et spécialisées liées à des groupes d'acteurs organisés sur le territoire qui donnent le ton des plaidoyers vertueux sur le service public et l'intérêt général. Les acteurs « sont pris dans la décision » davantage qu'ils ne la prennent. Les intérêts sociaux sont portés et masqués par les idéologies professionnelles, confortant la domination des élus locaux qui ignorent « avec une belle ostentation les clivages partisans ${ }^{14}$. Mais ces travaux font aussi le constat que « la gouvernance territoriale dessine une nouvelle cartographie de la domination politique, plus aléatoire que standardisée, plus asymétrique qu'uniforme mais bien réelle ${ }^{15}$.

Au cours de l'épisode des préconisations préfectorales, le « nous » politique des plaidoyers contre la réforme est souvent apparu en décalage complet avec les discours experts et professionnalisés, mais les débats ont aussi mis en évidence les dimensions aléatoires et asymétriques de ces prises de position. Le résultat le plus étonnant concerne sans doute le constat que les mots d'ordre vertueux des ingénieurs du développement local (le projet, les péréquations, l'intérêt communautaire, la qualité) semblaient avoir été démagnétisés au profit d'arguments beaucoup plus passionnels (le pays, les ressources, la démocratie, l'identité). Les orateurs ont en effet d'abord puisé leurs arguments dans le lexique des arguments sensibles à la qualité du «vivre ensemble » à petite échelle, à l'authenticité dans la proximité, à la solidarité dans la compassion. Autant de propos qui tenaient à distance les rhétoriques savantes sur l'intelligence territoriale pour leur préférer des formules empreintes de conviction (le sentiment d'appartenance), d'émotion (la fin d'une époque), voire d'indignation (la mondialisation sauvage). Ainsi, alors que la professionnalisation des administrations locales semblait annoncer, jusqu'à présent, la fin des idéologies territoriales et des clivages partisans, les enjeux de la réforme semblent avoir remis en selle des plaidoyers beaucoup plus substantiels que processuels. Pour l'observateur, il reste à savoir quels sont, dans la profusion des signes recueillis, ceux

12. Arnaud (Lionel), Le Bart (Christian), Pasquier (Romain), Idéologies et action publique territoriale. La politique change-t-elle encore les politiques?, Presses Universitaires de Rennes, Coll. Respublica, 2007, 257 p.

13. Pasquier (Romain), Simoulin (Vincent), Weisbein (Julien), La gouvernance territoriale. Pratiques, discours et théories, LGDJ, Droit et Société 44, 2007, 335 p.

14. Arnaud, Le Bart, Pasquier, op. cit., p. 251

15. Pasquier, Simoulin, Weisbein, op. cit., p. 222. 
qui dessinent éventuellement les contours de nouvelles perceptions de l'autorité et de la légitimité politiques. A titre prospectif, il nous semble possible de tester des hypothèses sur trois récits en particulier : la défiance métropolitaine, la souveraineté communale et le service public.

La première ligne narrative concerne les arguments de défiance identitaire que la thématique de la métropolisation cristallise immédiatement. Dès lors que leur commune n'est pas située dans une zone totalement urbanisée, les élus locaux vantent l'esprit des lieux sur les images idéaltypiques du village, de la ruralité, de la proximité, de l'authenticité, des interconnaissances, du terroir, de la culture locale, du bien être, de la solidarité, de la tranquillité. Ils plaident aussi le fait que les petites communes n'ont pas les mêmes « besoins » de services collectifs en raison de leur démographie. Et les propos établissent un parallèle immédiat avec une autre imagerie, résolument négative, qui serait attachée aux grands centres urbains, densifiés, hostiles, pollués, stressants... Ainsi, la «métropole», qui constitue la principale innovation sémantique dans la loi, attire presque mécaniquement les représentations diffuses d'un mal-vivre collectif lié à des problèmes récurrents en matière d'insécurité, de violence, de transports, d'environnement, de pauvreté. C'est à partir de cette même dichotomie symbolique que différents groupes organisés s'impliquent dans les débats sur la réforme. Les associations, sections locales de partis, syndicats et groupes d'usagers qui prennent publiquement position évoquent facilement la «menace » métropolitaine, l'impérialisme et l'arrogance du monde urbain, ses velléités évidentes d'annexion des territoires limitrophes et d'abandon des espaces ruraux de troisième couronne... Souvent, les conseils de développement qui ont souhaité donner une base argumentée à ces réflexions en organisant des réunions publiques ont favorisé des plaidoyers vibrants sur l'attachement indéfectible des habitants à l'identité communale et leur aversion profonde face aux désordres du monde urbain... Ainsi, au fil des débats, deux arguments tracent les frontières du clivage entre l'urbain et le rural : d'une part des récits qui insistent sur les ressorts traumatiques de l'identité communautaire (l'idée que les petites intercommunalités situées autour des agglomérations se sont construites et se fortifiées sur le reculoir, au gré d'événements douloureux, de blessures symboliques, de conflits territoriaux et d'échecs économiques qui ont imposé des solidarités défensives) ; d'autre part la description d'une métropolisation honteuse ${ }^{16}$ (l'idée que les grandes villes additionnent et condensent des problèmes de société et des disfonctionnements collectifs qui interdisent d'imaginer les facettes vertueuses et victorieuses de «l'air de la ville »).

La deuxième ligne narrative concerne l'attachement à une certaine forme de démocratie locale qui défend le principe de la souveraineté communale. Bien que le redimensionnement des redécoupages intercommunaux occupe une part importante des controverses, on peut aussi identifier une série d'arguments qui portent sur la façon dont les acteurs conçoivent les vertus démocratiques de la décentralisation. Les discours argumentent avec vigueur les mérites de la démocratie de proximité et donnent aux élus municipaux et aux habitants une place centrale dans les rouages de la décision publique. Ainsi en est-il (même si la loi l'interdit) des nombreux groupes d'usagers qui ont réclamé la mise en place d'un référendum local permettant aux habitants de se prononcer sur le rattachement de leur commune à une nouvelle intercommunalité (les prises de position sont nombreuses qui portent sur l'utilité et la nécessité des dispositifs de participation). Ainsi en est-il aussi des élus locaux, nombreux, qui ont dénoncé l'autoritarisme du diagnostic

16. Pour reprendre l'expression d'un président de communauté urbaine et de l'article titrant son intervention : Feltesse (Vincent), « La métropolisation honteuse », Esprit, mars-avril, 2011, p. 137-148. 
préfectoral, déplorant l'absence préalable de concertations et s'insurgeant contre le nouveau système de désignation et de représentativité des élus municipaux dans les arènes communautaires. Ainsi en est-il encore des acteurs qui plaident inlassablement le maintien des conseils généraux en raison de la sensibilité ruraliste de leurs élus, regrettant l'élargissement des découpages cantonaux et s'inquiétant des priorités détachées des « vrais problèmes » (trop éloignées des électeurs) que les conseillers communautaires et territoriaux risquent de favoriser. Toutes ces prises de position ont pour point commun de défendre la vision d'une souveraineté politique appuyée sur la représentation communale avec la conviction que la nouvelle donne électorale constitue une menace directe pour la démocratie locale.

La troisième ligne narrative concerne enfin l'équité du service public local, souvent argumentée sur des critères d'aménagement du territoire et de solidarité sociale. Les discours portent sur les missions de service public que la décentralisation doit encadrer et promouvoir. La réforme est l'occasion, pour différents acteurs ou groupes d'acteurs liés à des projets de développement local, de réaffirmer des principes directement inspirés par la doctrine aménagiste et planificatrice impulsée par l'État français depuis les années 1970. Les personnes qui sont par exemple impliquées dans des dispositifs locaux de maillage des territoires (pays, contrats, parcs, schémas, missions, antennes, services publics locaux...) argumentent une vision éthique du service public, de l'action sociale et de l'aménagement foncier qui repose sur les préceptes éprouvés de «l'État local ». Cette représentation défend l'idée que le système local a besoin de médiations publiques intermédiaires, c'est-à-dire placées à bonne distance tout à la fois des intérêts locaux et des injonctions ministérielles. Dans les récits, on constate que cette distance sous-tend une vision républicaine de l'intérêt général en phase avec l'éthique des agents de développement (qu'ils soient territoriaux ou d'État) mais qui s'en distingue sans doute par ses formes narratives moins démonstratives : au vocabulaire des bonnes recettes et des formules magiques (projet, partenariat, pôles d'excellence, égalité, droit commun) succèdent des rhétoriques plus pragmatiques (qualité, usagers, équilibre, justice sociale, consultation, solidarité). Ces visions du service public perçoivent les «fiefs politiques » (qu'ils soient communaux ou intercommunaux) avec circonspection et prudence. On trouve de nombreuses illustrations de cet état d'esprit dans les diagnostics proposés par les conseils de développement, par les structures de conseil en matière d'action sociale et d'urbanisme, et bien sûr au sein des équipes travaillant sur les schémas de cohérence territoriale. Les débats sensibles sur la question des futurs Plans locaux d'urbanisme intercommunaux constituent à cet égard un baromètre significatif de la place que cette conception de la planification parvient (ou non) à occuper dans chaque configuration territoriale.

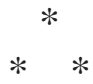

En cherchant à comprendre comment la politique se loge dans les activités discursives, notre brève incursion dans la sémantique des réactions à la réforme laisse entrevoir des indications sur la façon dont les énoncés construisent les références au pouvoir et à la démocratie locale. Les récits nous renseignent sur ce que la décentralisation signifie dans ses dimensions symboliques, idéologiques et passionnelles. Ils permettent aussi d'étudier les interactions qui relient l'illusio de la domination à la réalité des politiques publiques. En procédant à une lecture chronologique des débats, nous avons identifié des coalitions discursives qui participent de ce travail politique de construction et de mise en ordre des arrangements collectifs sur différents registres argumentaires. Certains sont en lien 
avec les luttes d'intérêts qui mobilisent les élus locaux dans leur conception du dialogue (vis-à-vis des communes voisines et du système politico-administratif départemental et régional) et dans les combats que le monde rural engage pour résister aux menaces et aux dangers de l'expansion urbaine. Nous avons aussi pointé des arguments qui relèvent plus des valeurs et des promesses de la politique, ses capacités de réenchantement en termes d'identité (fut-elle traumatique), de démocratie (fut-elle communaliste) et de service public (fut-il surplombant).

On peut considérer ces représentations comme des doctrines, des croyances et des stéréotypes qui encadrent, orientent et contraignent le jugement des acteurs dans leur appréhension des enjeux de gestion publique. Leur formation est puissamment balisée par des sentiers historiques qui perpétuent une conception guerrière et héroïque de la compétition politique locale tout en entretenant une vision républicaine surplombante du service public. La nouveauté, si nouveauté il y a, provient de l'ouverture des débats hors des cercles spécialisés de la décentralisation. C'est bien la première fois que la définition des périmètres d'action publique et de représentation politique faisait en France l'objet de controverses ouvertes et médiatisées dans chaque configuration territoriale. Dans ce contexte particulier, les élus locaux se sont trouvés placés dans une position des plus inconfortables : on leurs a demandé à la fois de changer de périmètre sans perdre la face et de changer de logiciel sans que les communes ne perdent leur âme. L'étude des arguments convoqués pour affronter cette dialectique suggère que la gestion symbolique relève d'un défi cornélien au sens théâtral et tragique du terme. Dans Le Cid, les points de vue se heurtaient à la morale et à la religion, mais aussi à l'honneur et à l'amour. Le choix du changement devient très problématique pour les maires, comme il le fut pour Don Rodrigue, dès lors que les intérêts et les valeurs sont publiquement menacés dans la passion de leurs contradictions. La séquence de la réforme territoriale que nous avons étudiée rappelle la place centrale de cette dynamique symbolique dans la modification du grand récit tocquevillien sur les libertés locales. 